\title{
Controlling Charge-Transport in Metal-Organic Frameworks: Contribution of Topological and Spin-State Variation on the Fe- Porphyrin Centered Redox Hopping Rate
}

\author{
Karan Maindan, Xinlin Li, Jierui Yu, and Pravas Deria*, \\ Department of Chemistry and Biochemistry, Southern Illinois University, 1245 Lincoln Drive, \\ Carbondale, Illinois 62901, United State.
}

\section{S1 MOF samples preparation}

All the MOF samples, both in free base and Fe-metallated form were synthesized following various literature reported solvothermal procedure. ${ }^{1}$ In these syntheses, $\mathrm{ZrOCl}_{2} .8 \mathrm{H}_{2} \mathrm{O}$ was used as $\mathrm{Zr}^{\mathrm{IV}}$ source, benzoic acid as modulator and $\mathrm{H}_{4} \mathrm{TCPP}\left(\mathrm{H}_{2}\right)$ or $\mathrm{H}_{4} \mathrm{TCPP}(\mathrm{Fe})$ as linker.

Metalation of freebase porphyrin MOFs: The free-base MOF samples were post-synthetically metalated using $\mathrm{FeCl}_{2} .4 \mathrm{H}_{2} \mathrm{O}$. A portion of $\mathrm{MOF}$ sample $(\sim 50 \mathrm{mg})$ was added a $\mathrm{FeCl}_{2} \cdot 4 \mathrm{H}_{2} \mathrm{O}$ (20 equivalents per $\mathrm{TCPPH}_{2}$ present in the MOF) solution in $5 \mathrm{~mL}$ DMF or acetonitrile under inert condition. This reaction mixture in a microwave-safe glass vial was heated in the microwave reactor for 3 hours at $120{ }^{\circ} \mathrm{C}$. After bringing this mixture to room temperature the solid sample was thoroughly washed via centrifuge (DMF, $5 \times 10 \mathrm{~mL}$ ) and activated with $0.5 \mathrm{~mL} 8 \mathrm{M} \mathrm{HCl}$ in $10 \mathrm{~mL}$ DMF solvent. 


\section{S1 MOF samples characterization}
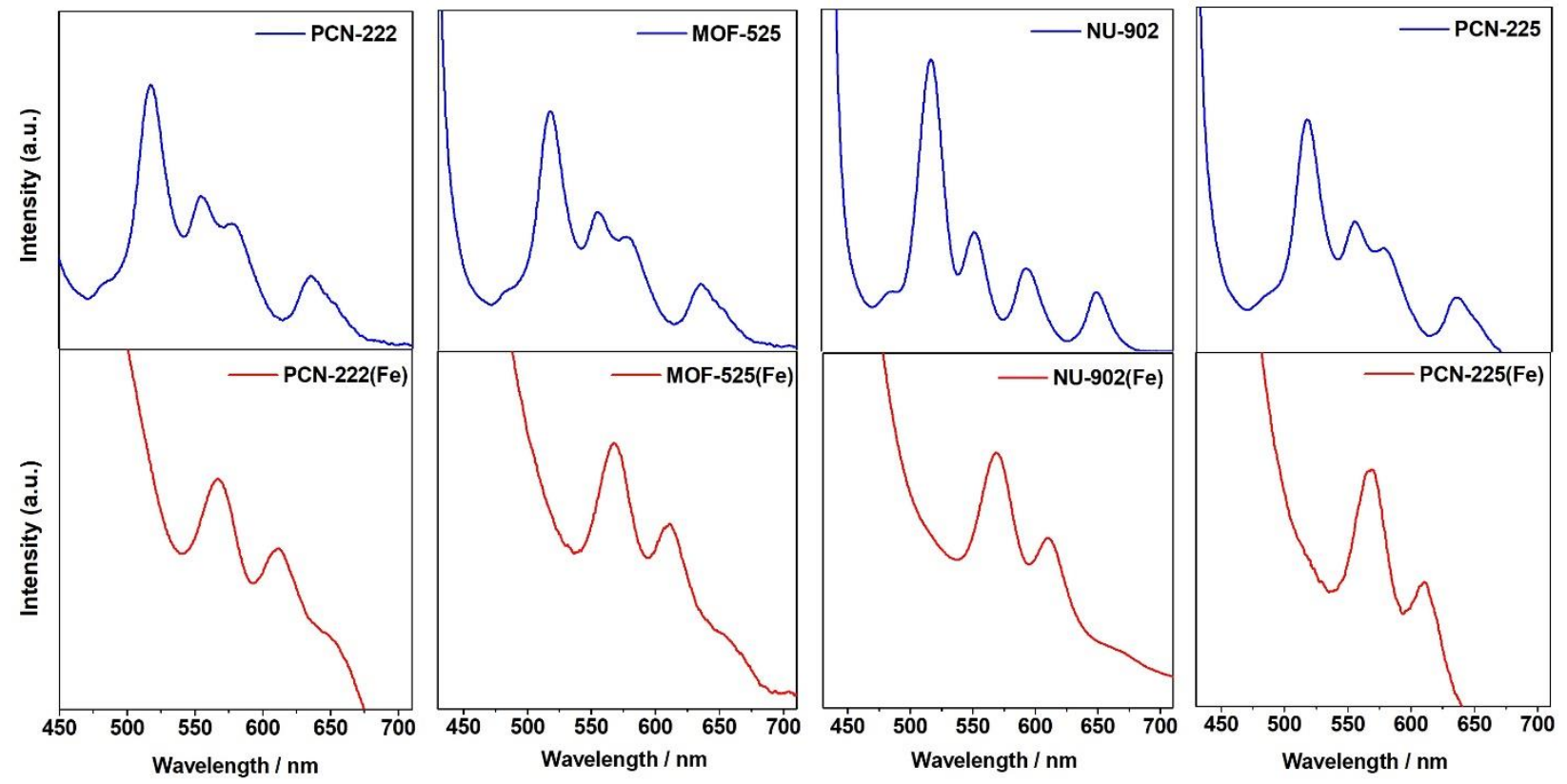

Figure S1. UV-Vis spectra of porphyrin-based MOFs (decomposed in $0.1 \mathrm{M} \mathrm{KOH}$ ) before and after metalation.

\begin{tabular}{|c|c|c|c|}
\hline & \multicolumn{2}{|c|}{ Experimental Atomic \% } & Expected Atomic \% \\
\hline Fe K & 0.9 & 1.11 & 1 \\
\hline Zr L & 2.45 & 3 & 3 \\
\hline
\end{tabular}
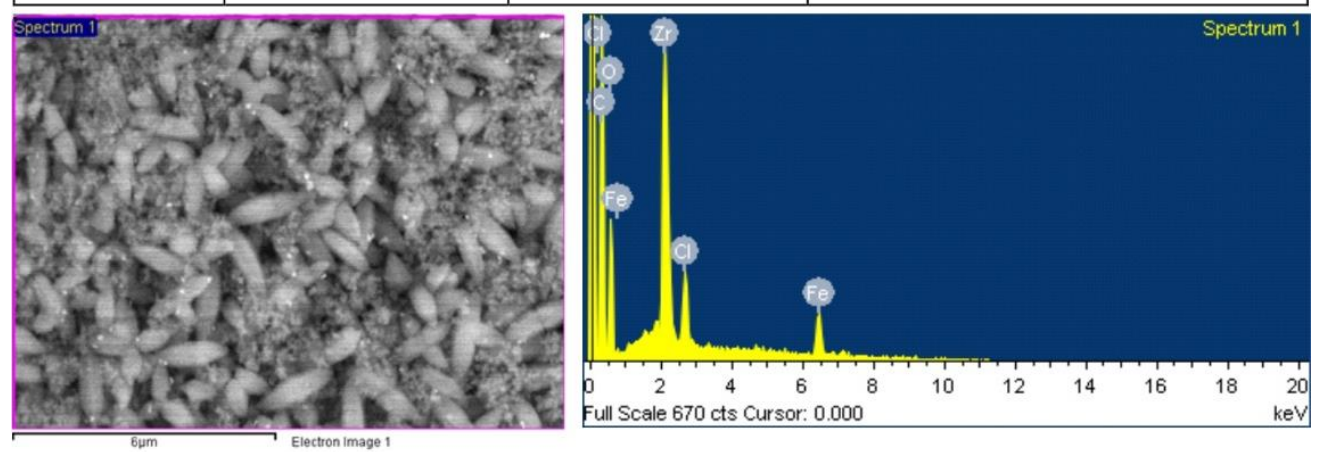

Figure S2. SEM-EDS spectrum of NU-902(Fe)/ PS-MOF 


\begin{tabular}{|c|c|c|c|}
\hline & \multicolumn{2}{|c|}{ Experimental Atomic \% } & Expected Atomic \% \\
\hline Fe K & 0.32 & 0.96 & 1 \\
\hline Zr L & 1 & 3 & 3 \\
\hline
\end{tabular}

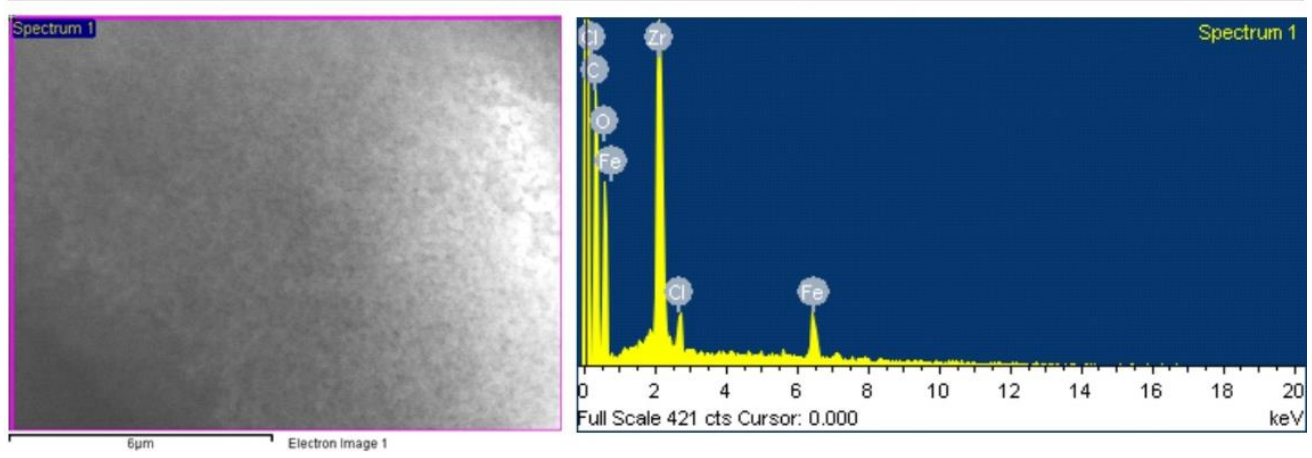

Figure S3. SEM-EDS spectrum of NU-902(Fe)/ DS-MOF

\begin{tabular}{|c|c|c|c|}
\hline & \multicolumn{2}{|c|}{ Experimental Atomic \% } & Expected Atomic \% \\
\hline Fe K & 1.14 & 1.17 & 1 \\
\hline Zr L & 2.96 & 3 & 3 \\
\hline
\end{tabular}

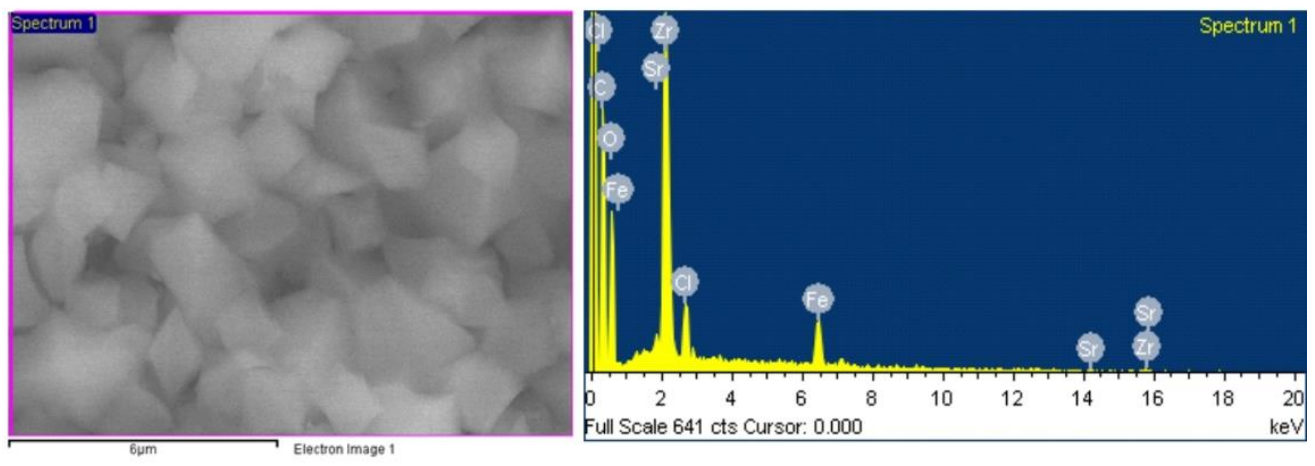

Figure S4. SEM-EDS spectrum of PCN-225(Fe)/ DS-MOF 


\begin{tabular}{|c|c|c|c|}
\hline & \multicolumn{2}{|c|}{ Experimental Atomic \% } & Expected Atomic \% \\
\hline Fe K & 0.38 & 1.14 & 1 \\
\hline Zr L & 1 & 3 & 3 \\
\hline
\end{tabular}

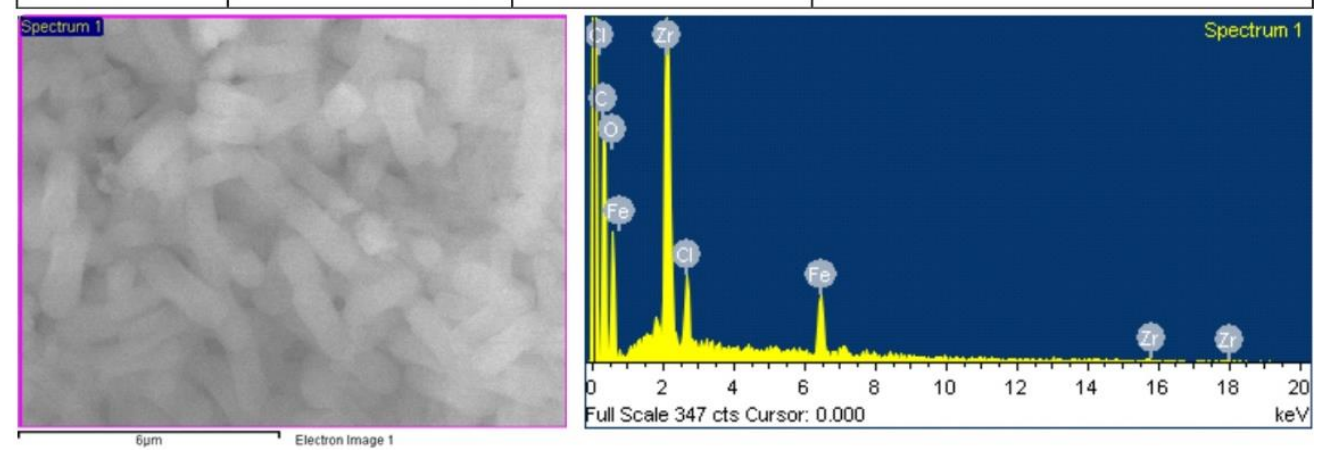

\begin{tabular}{|c|c|c|c|}
\hline & \multicolumn{2}{|c|}{ Experimental Atomic \% } & Expected Atomic \% \\
\hline Fe K & 0.52 & 1.04 & 1 \\
\hline Zr L & 1 & 2 & 2 \\
\hline
\end{tabular}

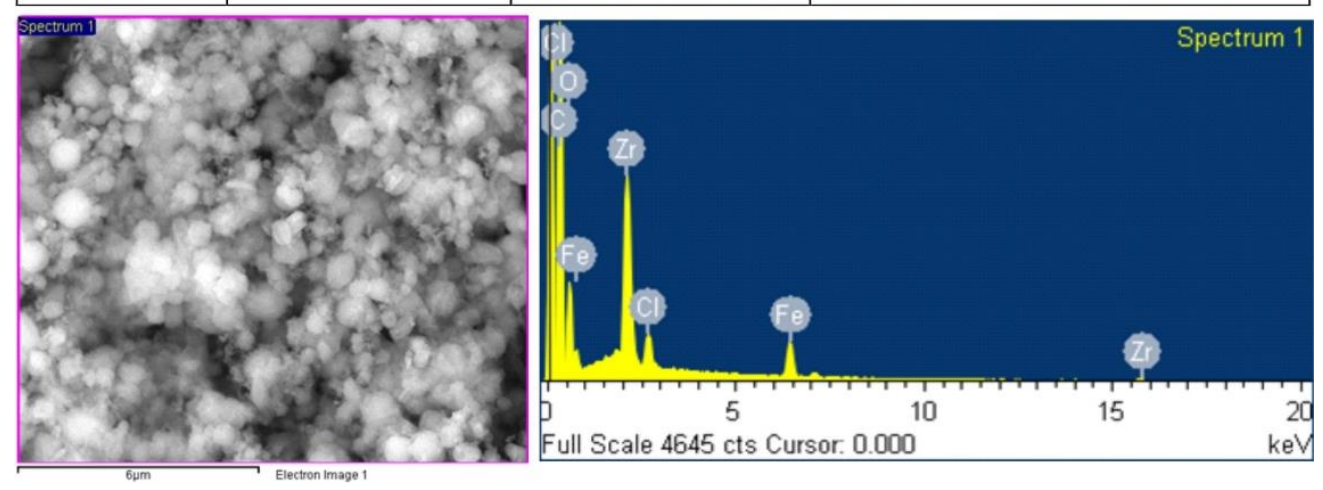

Figure S5. (Top) SEM-EDS spectrum of PCN-222(Fe)/ DS-MOF. (Bottom) SEM-EDS spectrum of MOF-525(Fe)/ DS-MOF 

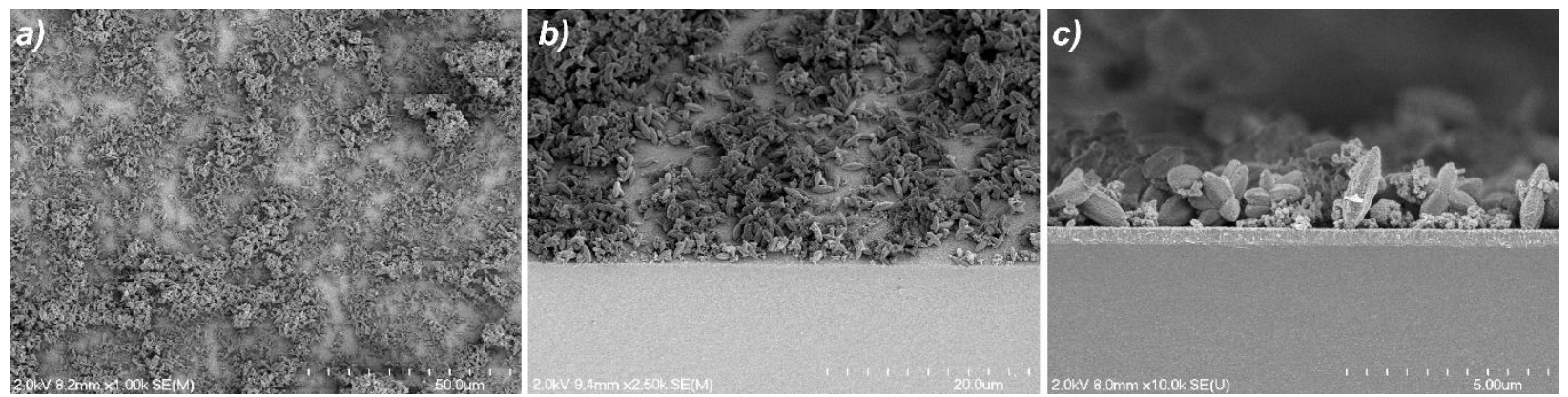

Figure S6. SEM images of a used NU-902(Fe) on FTO from $a$ ) top $b$ ) tilted at $45^{\circ}$, and $c$ ) side (note the small crystals of dried electrolyte). The morphology of the film is comparable to those reported in Usov, P. M. et.al. ACS Appl. Mater. Interfaces, 2017, 9, 33539-33543

\section{S3 Electrochemical studies of MOF-films}

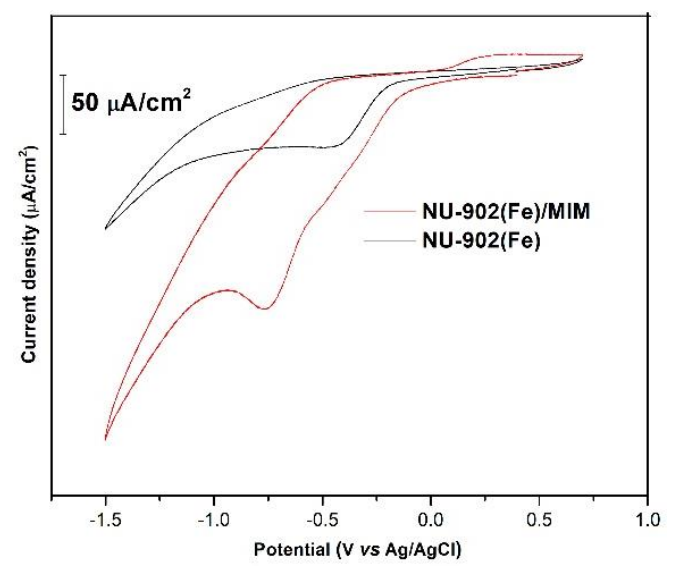

Figure S7. CV plots of NU-902(Fe) and NU-902(Fe)/MIM highlighting the $\mathrm{Fe}^{\mathrm{III} / \mathrm{II}}$ redox event. Experimental conditions: $1 \mathrm{M} \mathrm{TBAPF}_{6}$ in acetonitrile solvent; scan rate $=100 \mathrm{mV} / \mathrm{s}$. MOFs deposited (probed area $=1 \mathrm{~cm}^{2}$ ) FTO as working electrode.

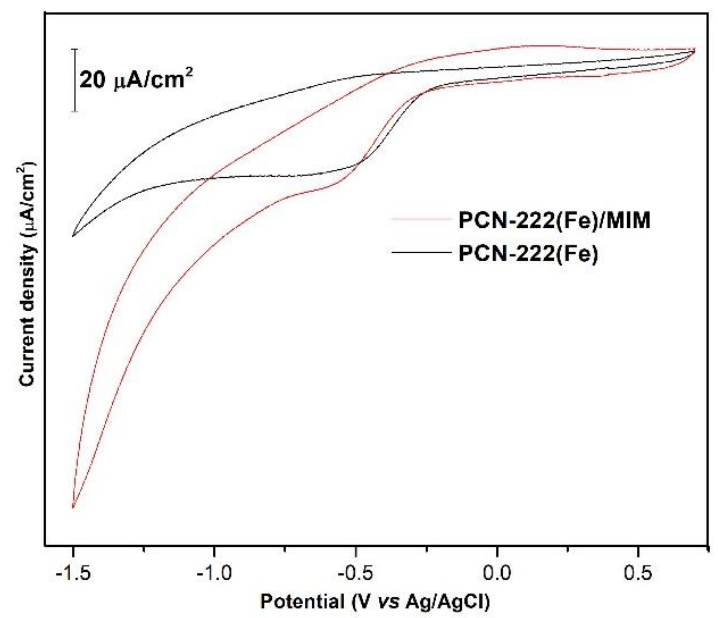


Figure S8. CV plots of PCN-222(Fe) and PCN-222(Fe)/MIM highlighting the $\mathrm{Fe}{ }^{\mathrm{III} / I I}$ redox event. Experimental conditions: $1 \mathrm{M} \mathrm{TBAPF}_{6}$ in acetonitrile solvent; scan rate $=100 \mathrm{mV} / \mathrm{s}$. MOFs deposited (probed area $=1 \mathrm{~cm}^{2}$ ) FTO as working electrode.
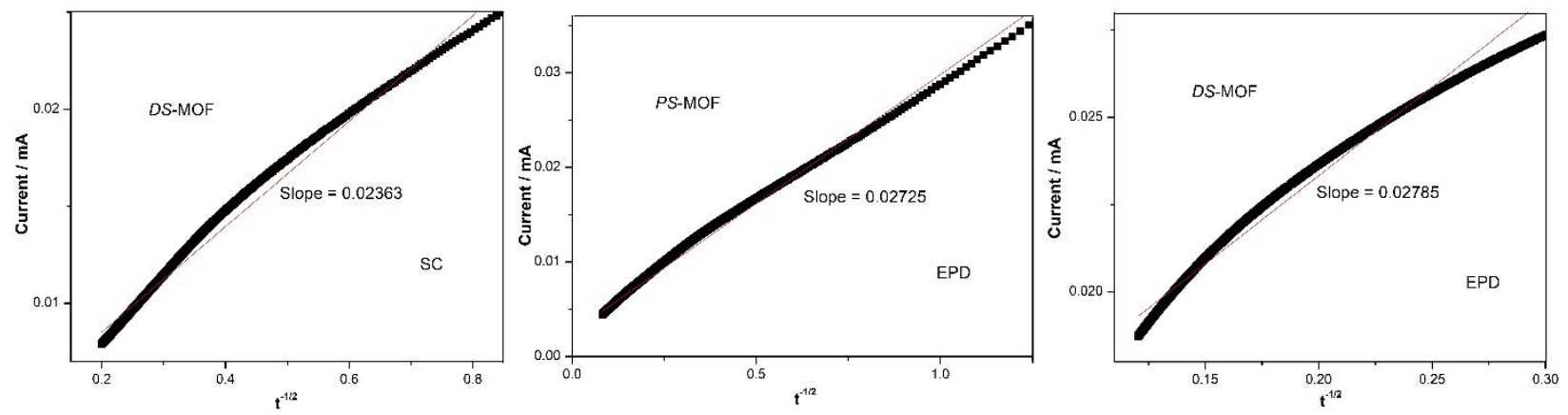

Figure S9. Cottrell plots of transient currents measured for PCN-222(Fe) during the Fe ${ }^{\mathrm{III} / \mathrm{II}}$ redox event. Experimental conditions: $1 \mathrm{M} \mathrm{TBAPF}_{6}$ in acetonitrile solvent; MOFs deposited (probed area $=1 \mathrm{~cm}^{2}$ ) FTO as working electrode.
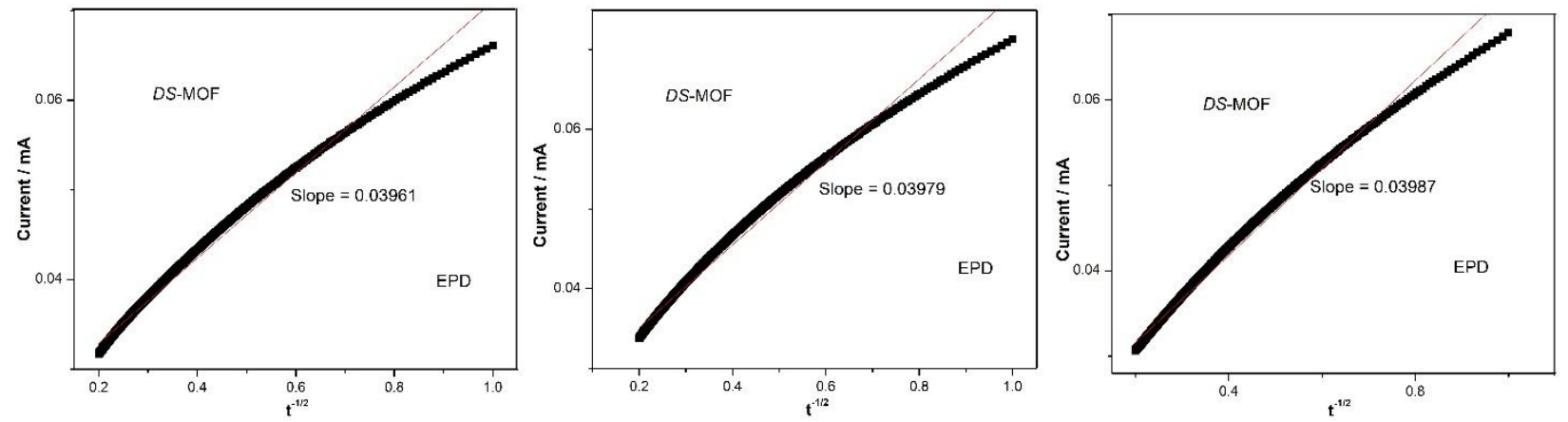

Figure S10. Cottrell plots of transient currents measured for PCN-222(Fe)/MIM during the Fe ${ }^{\mathrm{III} / \mathrm{II}}$ redox event. Experimental conditions: $1 \mathrm{M} \mathrm{TBAPF}_{6}$ in acetonitrile solvent; MOFs deposited (probed area $=1$ $\mathrm{cm}^{2}$ ) FTO as working electrode.
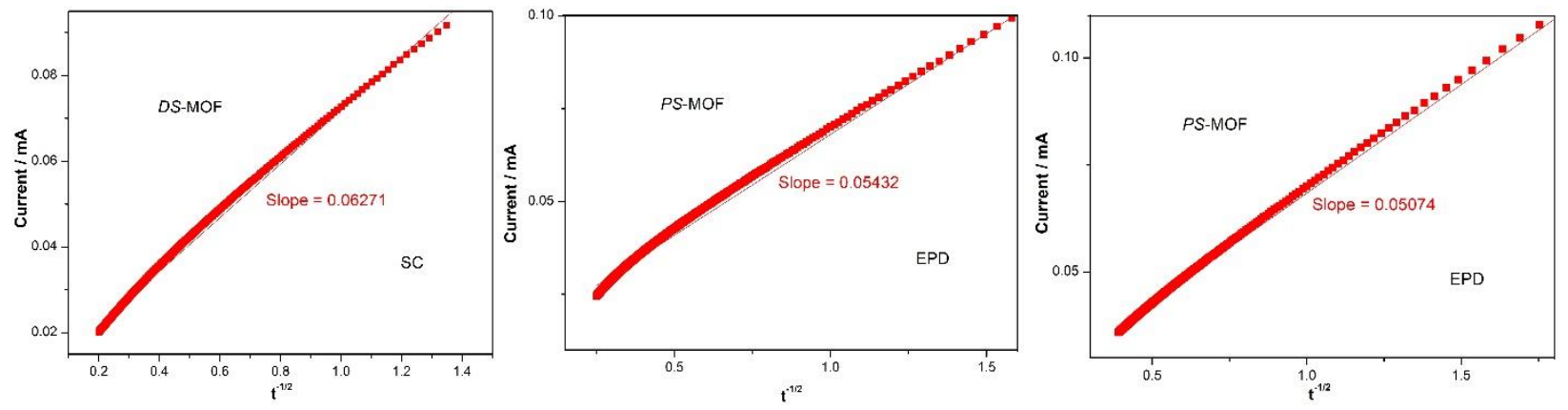

Figure S11. Cottrell plots of transient currents measured for MOF-525(Fe) during the $\mathrm{Fe}^{\mathrm{III} / \mathrm{II}}$ redox event. Experimental conditions: $1 \mathrm{M} \mathrm{TBAPF}_{6}$ in acetonitrile solvent; MOFs deposited (probed area $=1 \mathrm{~cm}^{2}$ ) FTO as working electrode. 

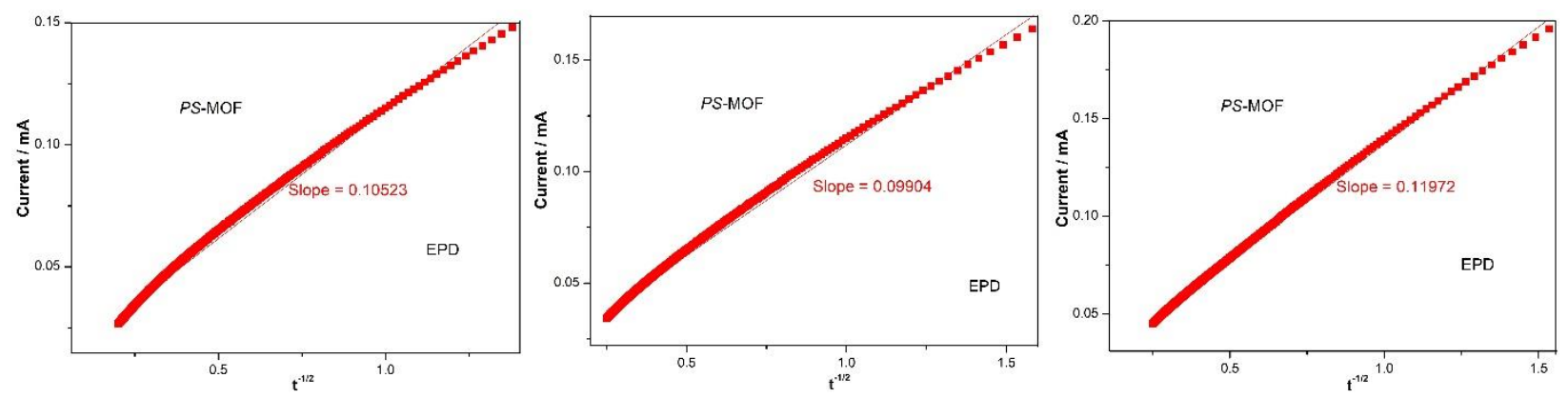

Figure S12. Cottrell plots of transient currents measured for MOF-525(Fe)/MIM during the $\mathrm{Fe}^{\mathrm{III} / \mathrm{II}}$ redox event. Experimental conditions: $1 \mathrm{M} \mathrm{TBAPF}_{6}$ in acetonitrile solvent; MOFs deposited (probed area $=1$ $\mathrm{cm}^{2}$ ) FTO as working electrode.
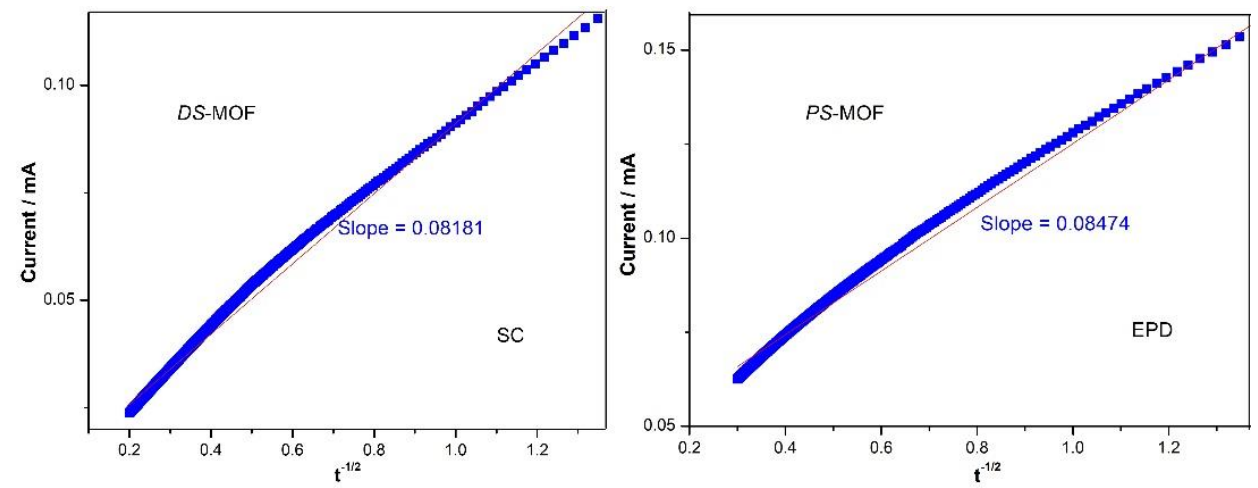

Figure S13. Cottrell plots of transient currents measured for NU-902( $\mathrm{Fe})$ during the $\mathrm{Fe}^{\mathrm{III} / \mathrm{II}}$ redox event. Experimental conditions: $1 \mathrm{M} \mathrm{TBAPF}_{6}$ in acetonitrile solvent; MOFs deposited (probed area $=1 \mathrm{~cm}^{2}$ ) FTO as working electrode.
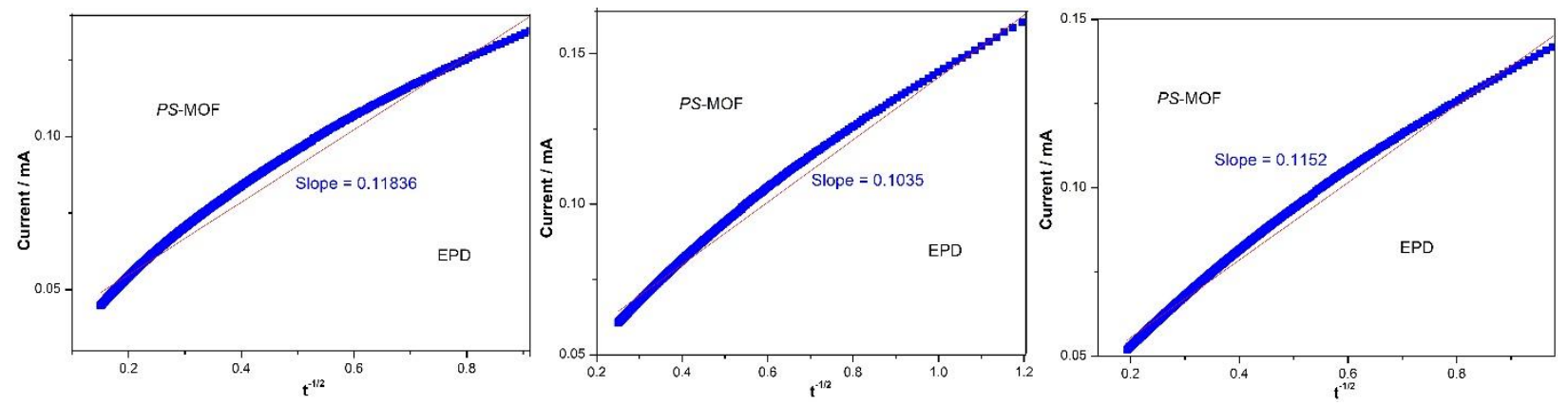

Figure S14. Cottrell plots of transient currents measured for NU-902(Fe)/MIM during the $\mathrm{Fe}^{\mathrm{III} / \mathrm{II}}$ redox event. Experimental conditions: $1 \mathrm{M} \mathrm{TBAPF}_{6}$ in acetonitrile solvent; MOFs deposited (probed area $=1$ $\mathrm{cm}^{2}$ ) FTO as working electrode. 

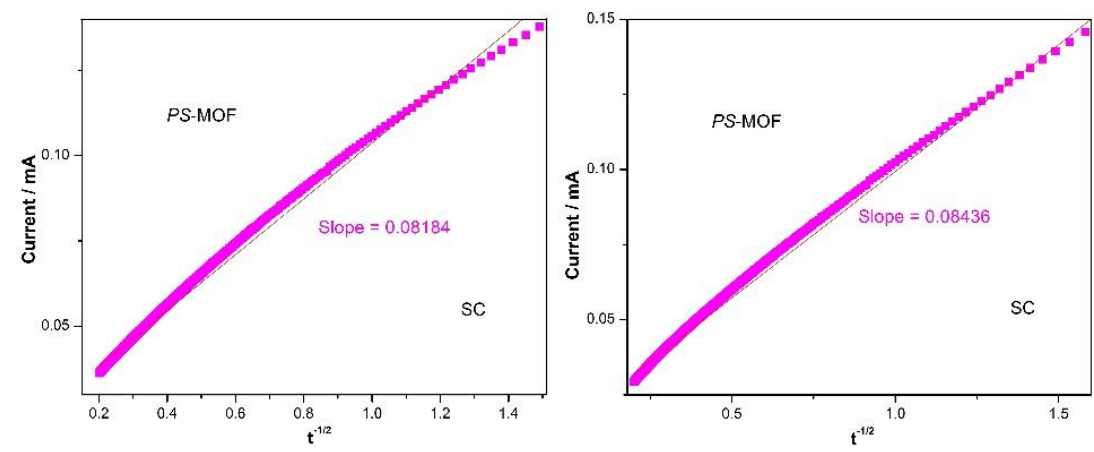

Figure S15. Cottrell plots of transient currents measured for PCN-225(Fe) during the $\mathrm{Fe}{ }^{\mathrm{III} / \mathrm{II}}$ redox event. Experimental conditions: $1 \mathrm{M} \mathrm{TBAPF}_{6}$ in acetonitrile solvent; MOFs deposited (probed area $=1 \mathrm{~cm}^{2}$ ) FTO as working electrode.

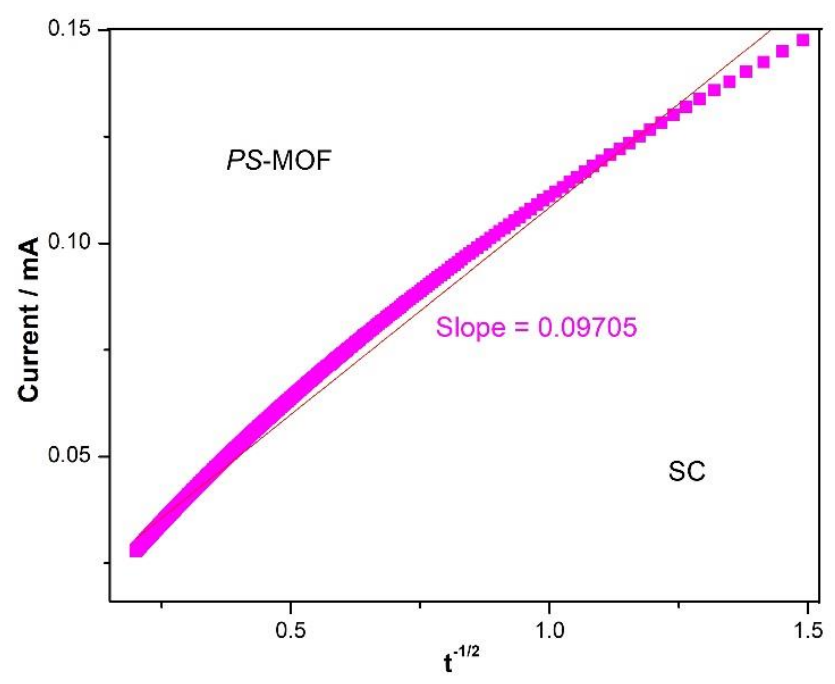

Figure S16. Cottrell plots of transient currents measured for PCN-225(Fe)/MIM during the Fe ${ }^{\mathrm{III} / \mathrm{II}}$ redox event. Experimental conditions: $1 \mathrm{M} \mathrm{TBAPF}_{6}$ in acetonitrile solvent; MOFs deposited (probed area $=1$ $\mathrm{cm}^{2}$ ) FTO as working electrode.
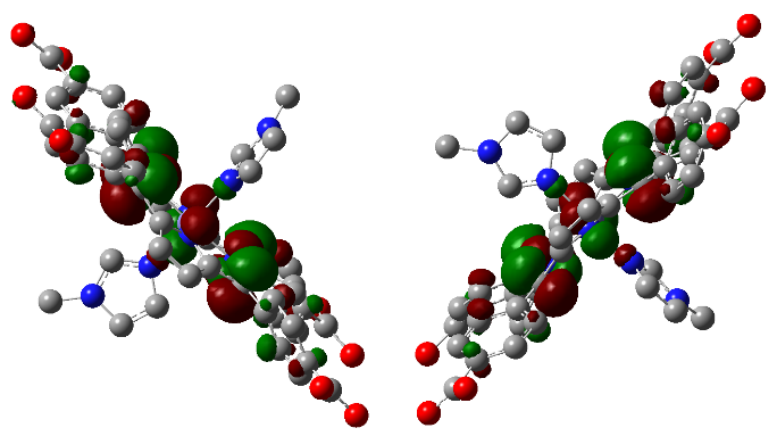

Figure S17. HOMO of MOF-525(Fe)/MIM. Method: CAM-B3LYP; Basis set: LANL2DZ 
1. (a) Jiang, H.-L.; Feng, D.; Wang, K.; Gu, Z.-Y.; Wei, Z.; Chen, Y.-P.; Zhou, H.-C., An Exceptionally Stable, Porphyrinic Zr Metal-Organic Framework Exhibiting pH-Dependent Fluorescence. J. Am. Chem. Soc. 2013, 135 (37), 13934-13938; (b) Deria, P.; Yu, J.; Balaraman, R. P.; Mashni, J.; White, S. N., Topology-dependent emissive properties of zirconium-based porphyrin MOFs. Chem. Commun. 2016, 52 (88), 13031-13034; (c) Kelty, M. L.; Morris, W.; Gallagher, A. T.; Anderson, J. S.; Brown, K. A.; Mirkin, C. A.; Harris, T. D., High-throughput synthesis and characterization of nanocrystalline porphyrinic zirconium metal-organic frameworks. Chem. Commun. 2016, 52 (50), 7854-7857; (d) Gong, X.; Noh, H.; Gianneschi, N. C.; Farha, O. K., Interrogating Kinetic versus Thermodynamic Topologies of MetalOrganic Frameworks via Combined Transmission Electron Microscopy and X-ray Diffraction Analysis. $J$. Am. Chem. Soc. 2019, 141 (15), 6146-6151; (e) Shaikh, S. M.; Usov, P. M.; Zhu, J.; Cai, M.; Alatis, J.; Morris, A. J., Synthesis and Defect Characterization of Phase-Pure Zr-MOFs Based on Mesotetracarboxyphenylporphyrin. Inorg. Chem. 2019, 58 (8), 5145-5153. 